\title{
Giant optical oscillator strengths in perturbed hexagonal germanium
}

\author{
Abderrezak Belabbes $^{1}$, Friedhelm Bechstedt ${ }^{2}$, and Silvana Botti ${ }^{2}$ \\ ${ }^{1}$ Department of Physics, Sultan Qaboos University, P.O. Box 36, PC 123, Muscat, Oman and \\ ${ }^{2}$ Institut für Festkörpertheorie and-optik, Friedrich-Schiller-Universität Jena, Max-Wien-Platz 1, 07743 Jena, Germany \\ (*abderrezak.belabbes@uni-jena.de)
}

\begin{abstract}
We present $a b$ initio calculations of electronic and optical properties of perturbed hexagonal germanium and demonstrate that it is a superior material for active optoelectronic devices in the infrared spectral region. It is known that perfect lonsdaleite $\mathrm{Ge}$ is a pseudodirect semiconductor, i.e., with direct fundamental band gap but almost vanishing oscillator strength for the lowest-energy optical transitions. Perturbing the system by replacing a $\mathrm{Ge}$ atom in the unit cell with a $\mathrm{Si}$ atom boosts of the oscillator strength at the minimum direct gap by orders of magnitude, with a concurrent blue shift of the interband distances. This effect is mainly due to the increased $s$ character of the lowest conduction band because of the perturbation-induced wave function mixing. A purely structural modification of the lonsdaleite unit cell of hexagonal Ge yields as well increased optical oscillator strengths, but their magnitude significantly depends on the actual details of the atomic geometry. In particular, moderate tensile uniaxial strain can induce an inversion of the order of the two lowest conduction bands, immediately leading to an extremely efficient enhancement of optical transitions. In general, chemical and/or structural perturbations of the lonsdaleite lattice are shown to be the key to make hexagonal germanium suitable for light emitting devices.
\end{abstract}

\section{INTRODUCTION}

Diamond silicon $(\mathrm{Si})$ is the key material of semiconductor industry. However, since it is an indirect band-gap semiconductor, it cannot emit light efficiently. Therefore, Si cannot be employed in active photonic devices or in optical intrachip communication. Modifications of this fundamental semiconductor are desirable to increase its light emission and absorption efficiency and make it suitable for optoelectronic applications [1, 2]. Mixing Si with germanium (Ge) keeps the compatibility of the resulting $\mathrm{Si}_{x} \mathrm{Ge}_{1-x}$ alloys with CMOS processing, but this modification still leads to indirect semiconductors. Only alloying in combination with a variation the crystal structure from cubic diamond (3C) to hexagonal lonsdaleite (2H) [3] allows for efficient light emission. This has been recently demonstrated for nanostructured hexagonal (hex) Ge-rich alloys [4].

First-principles electronic structure calculations for bulk lonsdaleite Ge [5] find that this material has a direct band gap at the Brillouin zone (BZ) center $\Gamma$ but with an extremely small oscillator strength associated to the optical transition from the valence band maximum (VBM) into the lowest conduction band minimum (CBM). As a consequence, $2 \mathrm{H}-\mathrm{Ge}$ can be classified as a pseudodirect semiconductor. Replacing a fraction of the Si atoms with Ge to obtain the alloy hex$\mathrm{Si}_{x} \mathrm{Ge}_{1-x}$, with the $2 \mathrm{H}$ bond pattern, induces a strong decrease by three orders of magnitudes of radiative lifetime until reaching about $50 \% \mathrm{Si}$ content [6]. The direct-indirect transition of the band gap character as a function of Si molar fraction $x$ has been recently addressed by several studies [6-9]. The optical properties of these alloys have been calculated from first principles, including quasiparticle and excitonic effects, for $\mathrm{Si}$ rich hexagonal alloys within the virtual crystal approximation [7] and for $2 \mathrm{H}-\mathrm{Ge}$ [8]. However, the quantum mechanical reasons of the forbidden optical transitions at the direct band gap of $2 \mathrm{H}-\mathrm{Ge}$ have remained unexplored. An explanation of how alloying in Ge-rich systems or perturbations of the atomic arrangements influence the onset transitions is still missing. As a result, there is a need to investigate how modifications of $2 \mathrm{H}-\mathrm{Ge}$ can transform it from a pseudodirect semiconductor to a direct band-gap material.

The drastic changes in the optoelectronic properties going from pure $2 \mathrm{H}-\mathrm{Ge}$ to $\mathrm{Ge}$-rich hex- $\mathrm{Si}_{x} \mathrm{Ge}_{1-x}$ alloys ask for a deeper microscopic understanding. To this end, in this Letter we calculate variations of the electronic band structure and compare optical matrix elements for dipole transitions with photon energies $\hbar \omega$ near the absorption edge for hexagonal alloys with about $25 \%$ silicon and pure $2 \mathrm{H}-\mathrm{Ge}$. More precisely, we consider the hexagonal four-atom cell and its BZ and investigate, besides pure lonsdaleite germanium, also the crystal obtained by replacing one $\mathrm{Ge}$ atom with a $\mathrm{Si}$ one. The latter atomic arrangement describes a hexagonal $\mathrm{Si}_{0.25} \mathrm{Ge}_{0.75}$ alloy with an almost homogeneous distribution of Si substitutions over the entire crystal. To distinguish between chemical and structural perturbations of the starting $2 \mathrm{H}-\mathrm{Ge}$ crystal, we perform calculations also for atomic arrangements of only Ge atoms but with the equilibrium geometry of hexagonal $\mathrm{Si}_{0.25} \mathrm{Ge}_{0.75}$. Finally, as an example of stronger structural perturbation, we consider the geometry obtained by applying $1.8 \%$ tensile uniaxial strain along the $c$-axis on $2 \mathrm{H}-\mathrm{Ge}$ and the resulting changes of the electronic structure.

\section{RESULTS AND DISCUSSIONS}

We optimized the considered hex-Ge and hex-SiGe cells and calculated the corresponding electronic and optical properties using density functional theory (DFT). The computational details are discussed in section IV.

The incorporation of $25 \%$ of $\mathrm{Si}$ induces a shrinking of the lattice in comparison with $2 \mathrm{H}-\mathrm{Ge}$, yielding for $\mathrm{Si}_{0.25} \mathrm{Ge}_{0.75}$ lattice constants of $a=3.946$ (3.996) $\AA$ and $c=6.155$ (6.592) $\AA$ with ratio $c / a=1.651(1.650)>\sqrt{8 / 3}$ and an internal-cell parameter $u=0.374(0.374) \lesssim 3 / 8$ (the values in parenthesis are those for $2 \mathrm{H}-\mathrm{Ge}$ [5]). The space group symmetry is lowered from $\mathrm{P}_{3} / \mathrm{mmc}\left(C_{6 v}^{4}\right)$ to $\mathrm{P} 3 \mathrm{~m} 1\left(C_{3 v}^{1}\right)$, accom- 
panied by a point group change from $6 / \mathrm{mmm}\left(D_{6 h}\right)$ to $\overline{6} \mathrm{~m} 2$ $\left(D_{3 h}\right)$. The $u$ parameter for hex- $\mathrm{Si}_{0.25} \mathrm{Ge}_{0.75}$ is an average of the values $u=0.370 / 0.378$ along the vertical Si-Ge/Ge-Ge bond in the unit cell. Symmetry-conserving uniaxial strain leads to lattice parameters $a=3.987 \AA, c=6.712 \AA$, and $u=0.372$. The elongation of the unit cell along the $c$-axis is accompanied by a shrinking of the cell in the perpendicular directions.

DFT calculations of Kohn-Sham band structures are known to drastically underestimate the interband transition energies and band gaps when semi-local exchange correlation (XC) functionals are employed [10]. In view of that, to simulate the quasiparticle (QP) corrections to the DFT band structure due to electron or hole excitations, we apply an improved XC functional by Tran and Blaha [11, 12]: the modified BeckeJohnson (MBJ) potential[13] with the correlation term in the local density approximation (LDA), called MBJLDA functional. This functional has been proved to lead to accurate QP band energies for semiconductors, and in particular for $\mathrm{Si}$ and Ge crystals [5, 6, 14-16].

For the purpose of illustrating the precision of the MBJLDA electronic energies, the Bloch bands at $\Gamma$ are also computed using another approximate QP method, namely the HSE06 hybrid functional of Heyd, Scuseria and Ernzerhof (HSE) [17, 18]. In Table I] we can observe a rather good agreement between the energies calculated within the two approximate $\mathrm{QP}$ methods for both hex-Si $\mathrm{S}_{0.25} \mathrm{Ge}_{0.75}$ and $2 \mathrm{H}-\mathrm{Ge}$. The differences of valence-band energies are smaller than $10 \mathrm{meV}$. Even in the case of unoccupied bands the maximum deviations for the alloy remain below $0.1 \mathrm{eV}$. The optical dipole-matrix elements are computed in the longitudinal gauge [19] and presented in terms of Bloch matrix elements $P_{c v}^{\alpha}(\mathbf{k})=\left\langle c \mathbf{k}\left|p_{\alpha}\right| v \mathbf{k}\right\rangle$ of the $\alpha$ th Cartesian component $p_{\alpha}$ of the momentum operator for vertical transitions between valence band states $|v \mathbf{k}\rangle$ and conduction band states $|c \mathbf{k}\rangle$ with energies $\varepsilon_{v}(\mathbf{k})(v=$ $c, v)$. Excitonic effects are neglected since they are known to be weak at the absorption onset of germanium, independently of the Ge polymorph, because of the strong dielectric screening [8, 20].

The QP band structures of hex- $\mathrm{Si}_{0.25} \mathrm{Ge}_{0.75}$ and of pure $2 \mathrm{H}-$ Ge with the lattice constants and the atomic positions of hex$\mathrm{Si}_{0.25} \mathrm{Ge}_{0.75}$ are displayed in the panels (a) and (b), respectively, of Figs. 11. The electronic bands are plotted as a function of the vector $\mathbf{k}$ along high-symmetry lines in the BZ, considering only a small region of the $\mathrm{BZ}$ around the $\Gamma$ point and zooming on the energy interval close to the direct band gap. We apply for clarity the symmetry classification of the Bloch states derived for lonsdaleite also to hex- $\mathrm{Si}_{0.25} \mathrm{Ge}_{0.75}$ and the distorted $2 \mathrm{H}-\mathrm{Ge}$ cells, despite their symmetry reduction. The corresponding energy levels and band dispersions, i.e., the effective masses, near $\Gamma$ are given in Table [ In the case of $2 \mathrm{H}-$ $\mathrm{Ge}$ the energies at $\Gamma$ perfectly agree with previous calculations using the same approach [5]. This statement holds for the characteristic parameters of the splittings of the $p$-like valence bands, the crystal-field splitting $\Delta_{c f}=0.27 \mathrm{eV}$ and the spin-orbit splittings $\Delta_{s o}^{\|}=0.28 \mathrm{eV}$ and $\Delta_{s o}^{\perp}=0.27 \mathrm{eV}$. The effective masses are however slightly increased, apart from those of $\Gamma_{7 \pm v}$ along $\Gamma \mathrm{M}$. This is due to the strong sensitiv- ity of the effective masses to the size of the $\mathbf{k}$-space volume around the $\Gamma$-point considered to perform the parabolic fit of the band dispersion. The bands in Fig. 1 b), calculated for pure Ge using the atomic positions of the hex-SiGe cell, lead to only small variations with respect to the band structure of $2 \mathrm{H}-\mathrm{Ge}$. This especially holds for the valence bands. The conduction bands are slightly shifted toward higher energies as a consequence of the structural perturbation of the lonsdaleite lattice. Considering the reduction of the lattice constants, our findings are in qualitative agreement with results for a hydrostatic volume decrease shown in Ref. [21].

The largest shift happens for the second conduction band. More precisely, the changes of the atomic positions induce band shifts of $0.311\left(\Gamma_{7 c}\right), 0.061\left(\Gamma_{8 c}\right), 0.000\left(\Gamma_{9 v}\right),-0.010$ $\left(\Gamma_{7+v}\right)$, and $-0.032\left(\Gamma_{7-v}\right)$ eV with respect to $2 \mathrm{H}-\mathrm{Ge}$ (see Table II. Totally different bands are instead obtained for uniaxially strained hex-Ge (1.8\% tensile strain). In particular, this specific cell deformation leads to an inversion of the $\Gamma_{7 c}$ and $\Gamma_{8 c}$ conduction bands as visible in Fig. 2. This result is in agreement with earlier predictions [21]. The resulting band energies are $0.358\left(\Gamma_{7 c}\right), 0.421\left(\Gamma_{8 c}\right), 0.000\left(\Gamma_{9 v}\right)$, $0.138\left(\Gamma_{7+v}\right)$, and $-0.523 \mathrm{eV}\left(\Gamma_{7-v}\right)$. Because of the pure $s$ character of the $\Gamma_{7 c}$ state, its energy drastically moves down toward the VBM, while the $p$-containing $\Gamma_{8 c}$ conduction band only slightly shifts toward higher energies. This inversion of the two lowest conduction bands is in agreement with results of other strain studies for $2 \mathrm{H}-\mathrm{Ge}$ [22, 23] and also with the indirect-direct transition in 3C-Ge under tensile uniaxial strain [21].

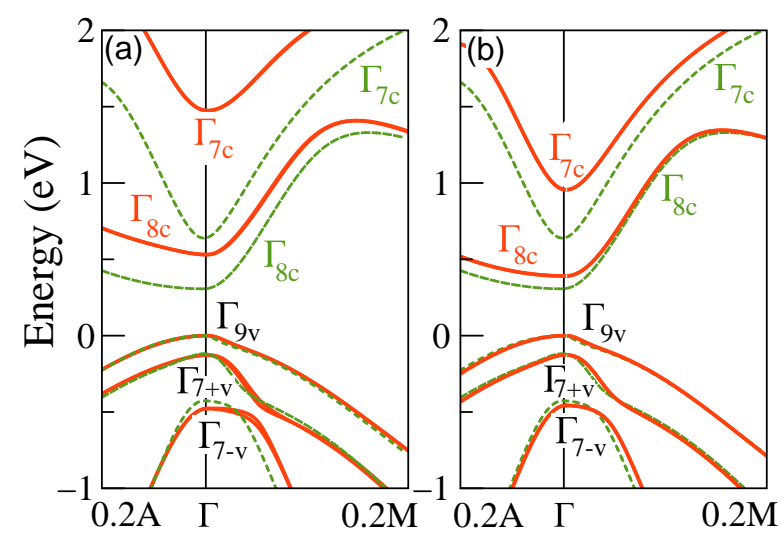

FIG. 1. Band structures around the $\Gamma$ point (red solid lines) of (a) hexagonal $\mathrm{Si}_{0.25} \mathrm{Ge}_{0.75}$ and (b) structurally perturbed $\mathrm{Ge}$ - arranged in the atomic geometry of optimized hexagonal $\mathrm{Si}_{1} \mathrm{Ge}_{3}$ - versus $\mathbf{k}$ points along high-symmetry lines in the hexagonal BZ. The bands are compared with the bands (green dashed lines) of pure $2 \mathrm{H}-\mathrm{Ge}$. The uppermost $\Gamma_{9 v}$ valence band is used as energy zero

. The band classification of the lonsdaleite symmetry group is also applied for the alloyed and structurally perturbed hex-Ge.

Mixing Si with Ge, in this case replacing $25 \%$ of the Ge atoms in the hexagonal cell, tends to increase significantly the band distances (see Fig. 11(a)), in agreement with the reduction of the atomic size from the covalent radius of $1.2 \AA$ $(\mathrm{Ge})$ to $1.11 \AA$ ( $\mathrm{Si})$ [24]. The resulting structural modifica- 
TABLE I. Modification of band distances and band dispersion near $\Gamma$ in hex-Si $i_{0.25} \mathrm{Ge}_{0.75}$ with respect to $2 \mathrm{H}-\mathrm{Ge}$ (values given in parenthesis). The state labels of pure $2 \mathrm{H}-\mathrm{Ge}$ are applied. The valence-band maximum is used as energy zero. The band energies computed with the HSE06 functional are listed in addition for the purpose of comparison. Three high-symmetry directions between the BZ center and boundary perpendicular $(\Gamma \mathrm{M}, \Gamma \mathrm{K})$ and parallel $(\Gamma \mathrm{A})$ to the $c$-axis are chosen to represent effective band masses. All effective masses are given in units of the free-electron mass $m$.

\begin{tabular}{|c|c|c|c|c|c|}
\hline \multirow[b]{2}{*}{ State } & \multicolumn{2}{|c|}{ position $(\mathrm{eV})$} & \multicolumn{3}{|c|}{ effective mass $(m)$} \\
\hline & MBJLDA & HSE06 & $\Gamma \rightarrow \mathrm{M}$ & $\Gamma \rightarrow \mathrm{K}$ & $\Gamma \rightarrow \mathrm{A}$ \\
\hline$\Gamma_{7 c}$ & $1.473(0.639)$ & $1.559(0.641)$ & $0.126(0.055)$ & $0.132(0.059)$ & $0.068(0.048)$ \\
\hline$\Gamma_{8 c}$ & $0.530(0.306)$ & $0.561(0.295)$ & $0.094(0.093)$ & $0.099(0.095)$ & $0.362(1.098)$ \\
\hline$\Gamma_{9 v}$ & $0.000(0.000)$ & $0.000(0.000)$ & $0.155(0.092)$ & $0.311(0.233)$ & $0.548(0.516)$ \\
\hline$\Gamma_{7+v}$ & $-0.127(-0.118)$ & $-0.138(-0.127)$ & $0.088(0.072)$ & $0.087(0.064)$ & $0.250(0.120)$ \\
\hline$\Gamma_{7-v}$ & $-0.477(-0.427)$ & $-0.486(-0.451)$ & $0.495(0.242)$ & $0.419(0.226)$ & $0.065(0.048)$ \\
\hline
\end{tabular}

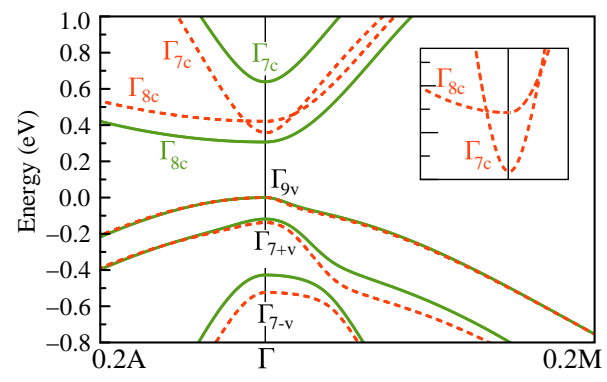

FIG. 2. Band structure of uniaxially strained $2 \mathrm{H}-\mathrm{Ge}$ (red dashed lines) in comparison with the unstrained crystal (green solid lines). The inset illustrates the band inversion at $\Gamma$.

tion may be interpreted as a compressive internal hydrostatic pressure of about $2.3 \%$ on the Ge lattice which opens the gap and interband distances. While this effect is small on the valence bands, the energy distances between conduction bands and their distances to the VBM are significantly increased, as it can be observed in Fig. 1 (a) and Table I] The comparison with the bands of pure $2 \mathrm{H}-\mathrm{Ge}$ with atoms arranged as in hex$\mathrm{Si}_{0.25} \mathrm{Ge}_{0.75}$, shown in Fig. 1. (b), proves that the valence bands are negligibly affected by this simple structural perturbation. However, the conduction-band shifts toward higher energies are significantly enhanced by chemical perturbation, i.e., the replacement of a $\mathrm{Ge}$ atom by a $\mathrm{Si}$ one. The fundamental gap $E_{g}=\Gamma_{8 c}-\Gamma_{9 v}$ is opened from 0.31 to $0.53 \mathrm{eV}$. The splitting $\Gamma_{7 c}-\Gamma_{8 c}$ of the two lowest conduction bands dramatically increases from 0.33 to $0.94 \mathrm{eV}$. The gap opening is in agreement with photoluminescence (PL) measurements [4] that indicate a shift of the PL peak position from $0.35 \mathrm{eV}$ for pure $\mathrm{Ge}$ to $0.67 \mathrm{eV}$ for $\mathrm{Si}_{0.35} \mathrm{Ge}_{0.65}$ in strong correlation with the fundamental gap variation in Table I. The wavelength $\lambda=3.54 \mu \mathrm{m}$ corresponding to the fundamental gap of $2 \mathrm{H}-\mathrm{Ge}$ is decreased to $1.85 \mu \mathrm{m}$ in hex- $\mathrm{Si}_{0.25} \mathrm{Ge}_{0.75}$. Incorporation of additional $\mathrm{Si}$ atoms may further reduce this wavelength toward the communication wavelength of $1.55 \mu \mathrm{m}$. The corresponding gap $E_{g} \approx 0.8 \mathrm{eV}$ should be reached for $\mathrm{Si}_{x} \mathrm{Ge}_{1-x}$ with a composition $x \approx 0.56$ applying linear gap interpolation. Similar values have been predicted elsewhere [4, 6].

The modifications of band dispersion in Fig. 1, described around $\Gamma$ by the effective masses in Table $\mathrm{I}$, do not show a unique trend with the incorporation of Si into the Ge lattice. The uppermost conduction band $\Gamma_{7 c}$ and the three valence bands $\Gamma_{9 v}, \Gamma_{7+v}$, and $\Gamma_{7-v}$ exhibit an increase of the effective masses. Only the lowest conduction band $\Gamma_{8 c}$ maintains its dispersion along the direction perpendicular to the $c$-axis but the effective electron mass parallel to the $c$-axis is reduced. This behavior is probably related to the increasing repulsion between the two lowest conduction bands with rising $x$, which also explains the increase of the energy distance $\Gamma_{7 c}-\Gamma_{8 c}$.

Without considering modifications in the band occupation, optical spectra $I^{\alpha}(\omega)$ such as absorption or luminescence are determined by expressions of the type

$$
\begin{array}{r}
I^{\alpha}(\omega) \sim \frac{1}{V} \sum_{\mathbf{k}} \sum_{c=\Gamma_{7 c}, \Gamma_{8 c} v=\Gamma_{9 v}, \Gamma_{7+v}, \Gamma_{7-v}}\left|P_{c v}^{\alpha}(\mathbf{k})\right|^{2} \\
\times \delta\left(\hbar \omega-\varepsilon_{c}(\mathbf{k})+\varepsilon_{v}(\mathbf{k})\right)
\end{array}
$$

for light-polarization direction $\alpha$ and photon energy $\hbar \omega$. The optical transition matrix elements $\left|P_{c v}^{\alpha}(\mathbf{k})\right|$ are plotted in Fig. 3 for all direct transitions between the three highest valence bands and the two lowest conduction bands along the highsymmetry directions $\Gamma \mathrm{M}$ and $\Gamma A$ (see Fig. 1) in pure lonsdaleite $\mathrm{Ge}$, hexagonal $\mathrm{Si}_{0.25} \mathrm{Ge}_{0.75}$ alloy and, for comparison, in hex-Ge with atomic positions determined by the alloy geometry. In Fig. 4 we present the optical transition matrix elements for hex-Ge under a tensile uniaxial strain of $1.8 \%$ with respect to the ideal lonsdaleite geometry.

In the case of lonsdaleite Ge the curves in Fig. 3 agree with previous results [5]. At $\Gamma$ they respect the selection rules imposed by group theory [25]. All transitions from the $p$-like valence bands into the $s$-like second conduction band $\Gamma_{7 c}$ in Fig. 3(b) are allowed, independently of the light polarization direction. Only for light polarized parallel to the $c$-axis, optical transitions from the uppermost valence band $\Gamma_{9 v}$ are forbidden. The situation is completely different for optical transitions involving the lowest conduction band $\Gamma_{8 c}$, as it can be observed in Fig.3 (3). All transitions at $\Gamma$ are dipole-forbidden. The only exception is the transition $\Gamma_{9 v} \rightarrow \Gamma_{8 c}$ for light polarization parallel to the $c$-axis in agreement with group theory [25]. However, the optical strength of the latter transition is by orders of magnitude, more precisely a factor of about 70 , smaller than that of the $\Gamma_{9 v} \rightarrow \Gamma_{7 c}$ transition. The origin of this disappointing result can be understood within the band- 


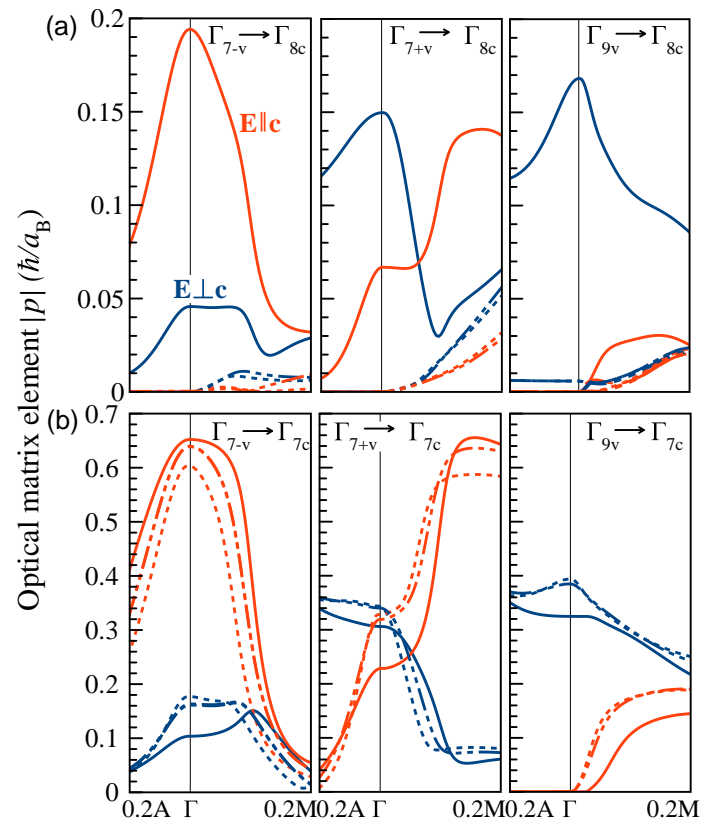

FIG. 3. Dipole transition matrix elements for optical transitions between the three highest valence bands $\Gamma_{9 v}, \Gamma_{7+v}$ and $\Gamma_{7-v}$ and (a) the first lowest conduction band $\Gamma_{8 c}$, as well as (b) the second lowest conduction band $\Gamma_{7 c}$ for the hex- $\mathrm{Si}_{0.25} \mathrm{Ge}_{0.75}$ alloy (solid lines), lonsdaleite $\mathrm{Ge}$ (dotted lines) and the structurally perturbed hexagonal $\mathrm{Ge}$ (dot-dashed lines). The curves are blue (red) for light polarization perpendicular (parallel) to the $c$-axis.

folding picture: the lowest conduction band of diamond $\mathrm{Ge}$ at two $L$ points is folded onto the $\Gamma$ point in the hexagonal BZ. Despite the modification of the atomic stacking in the hexagonal lattice compared with the diamond one, the strong $s p$-character of the $\Gamma_{8 c}$ states together with the accompanying vanishing oscillator strength between $p$-type valence and conduction bands is mainly conserved. The orbital character of the lowest conduction band is illustrated by orbital and site projections of the $\Gamma_{8 c}$ wave function in Table III Each of the four $\mathrm{Ge}$ atoms in the lonsdaleite cell contribute equally and show a $70 \%, 20 \%, 10) \%$ orbital character of $s . p_{z}, d_{z^{2}}$-type, respectively.

Modifying pure $2 \mathrm{H}-\mathrm{Ge}$ by replacing one $\mathrm{Ge}$ atom with $\mathrm{Si}$ to obtain hex-Si $\mathrm{Si}_{0.25} \mathrm{Ge}_{0.75}$, as shown in Fig. 3 b), leads to only small variations of the optical matrix elements of the transitions from the valence bands to the second-lowest $\Gamma_{7 c}$ conduction band. The situation is totally different for transitions into the lowest $\Gamma_{8 c}$ conduction band in Fig. 3 a). Apart from the $\Gamma_{9 v} \rightarrow \Gamma_{8 c}$ band transition with light polarization parallel to the $c$-axis, strong optical transitions appear in the alloyed system. Their dipole strengths are only smaller by factors of 2-3 compared to transitions to the $\Gamma_{7 c}$ band. Thereby the averaged $s$ contribution to the conduction band wave function is slightly increased (see Table II). However, the four atoms in the $\mathrm{Si}_{1} \mathrm{Ge}_{3}$ unit cell give rise to drastically different contributions to the total wave function. According to Table III, when the two Ge atoms lie above each other, bonded along the $c$ axis, the $s$-character of the " $\Gamma_{8 c}$ " wave function considerably

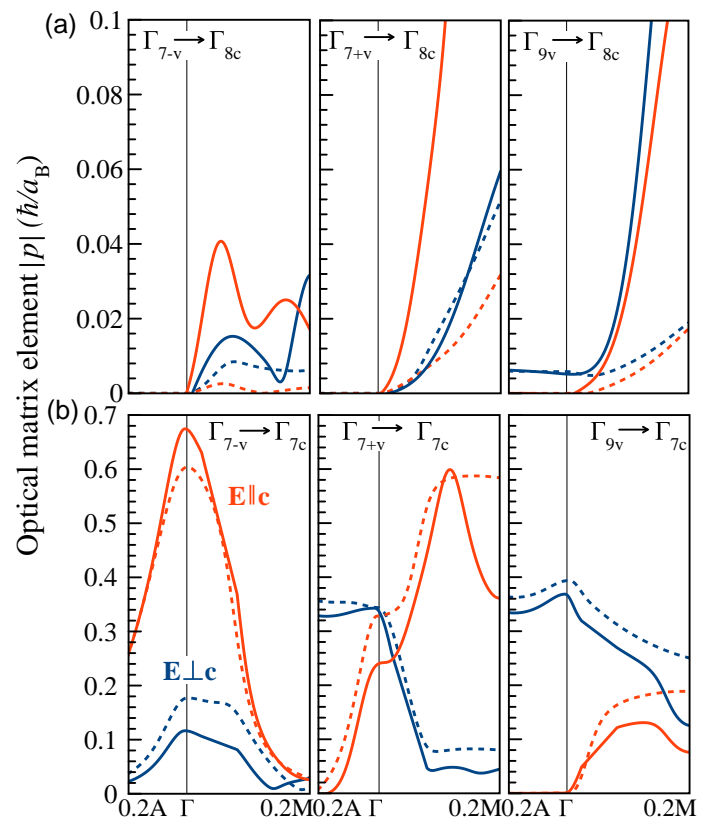

FIG. 4. Dipole transition matrix elements as in Fig. 3 but for hex-Ge under a tensile strain of $1.8 \%$ (solid lines), compared with those of unstrained $2 \mathrm{H}-\mathrm{Ge}$ (dotted lines).

increases up to $92 \%$. Therefore strong intra-atomic contributions to the oscillator strengths are possible. Small lattice perturbations, such as due to the substitution of one $\mathrm{Ge}$ atom in lonsdaleite by an isoelectronic $\mathrm{Si}$ atom together with the accompanying symmetry reduction of the atomic positions, internal strain and stronger mixing-in of $s$-orbitals into the lowest conduction band induce drastic changes in the optical oscillator strengths. The lowest optical transition $\Gamma_{9 v} \rightarrow \Gamma_{8 c}$ becomes really dipole-allowed for in-plane polarization with a giant strength similar to that in typical optoelectronic materials like GaAs [26].

TABLE II. Normalized orbital character of the lowest conduction band states $\Gamma_{8 c}$ characterized by the orbital- and site-projected character of the wave function for hex- $\mathrm{Si}_{0.25} \mathrm{Ge}_{0.75}, 2 \mathrm{H}-\mathrm{Ge}$, and structurally perturbed hex-Ge. In the case of hex- $\mathrm{Si}_{0.25} \mathrm{Ge}_{0.75}$ the fourth atom is $\mathrm{Si}$.

\begin{tabular}{cccc}
\hline \hline & \multicolumn{3}{c}{ orbital } \\
\cline { 2 - 4 } atom & $s$ & $p_{z}$ & $d_{z^{2}}$ \\
\hline 1 & $0.92,0.70,0.71$ & $0.02,0.20,0.13$ & $0.06,0.10,0.16$ \\
2 & $0.83,0.70,0.65$ & $0.14,0.20,0.24$ & $0.03,0.10,0.10$ \\
3 & $0.51,0.70,0.75$ & $0.24,0.20,0.13$ & $0.26,0.10,0.12$ \\
4 & $0.41,0.70,0.70$ & $0.48,0.20,0.21$ & $0.12,0.10,0.09$ \\
average & $0.75,0.70,0.71$ & $0.17,0.20,0.18$ & $0.09,0.10,0.11$ \\
\hline \hline
\end{tabular}

It is possible that structural and chemical perturbations may explain why in nanostructured Ge core-shell wires strong emission is experimentally observed already for nominally pure hexagonal Ge. Similar theoretical and experimental observations have been made for pseudodirect wurtzite semiconductors in comparison to indirect zinc-blende materials, e.g. for InP nanowires alloyed with $\mathrm{Al}$ [27], or in wurtzite $\mathrm{GaP}$ 
nanowires, where strong many-body excitonic effects or tensile uniaxial strain may explain the measured strong luminescence [28-30].

For a better understanding of the origin of the increased oscillator strengths we have separately considered the effect of a purely structural perturbation versus combined chemical and structural perturbation on lonsdaleite Ge. Considering the results in Fig. 3 and Table II], we can conclude that the influence of the structural perturbation is much weaker than the chemical effect, despite the fact that $\mathrm{Si}$ and $\mathrm{Ge}$ are isovalent. The structural relaxation increases the interband transition energies in Fig. 1.b) and the oscillator strengths in Fig. 3. However, the effects are smaller as in the case of a concurrent chemical and structural modification, as in the hexagonal alloy $\mathrm{Si}_{0.25} \mathrm{Ge}_{0.75}$. The small changes of the momentum matrix elements are thereby in line with the minor variation of the orbital character in Table II. The small effect of purely structural modifications cannot be generalized. In the case of moderate tensile uniaxial strain, as shown for $2 \mathrm{H}-\mathrm{Ge}$ in Fig. 4, we can still report weak or moderate changes of the dipole strengths of the transitions $\Gamma_{9 v}, \Gamma_{7+v}, \Gamma_{7-v} \rightarrow \Gamma_{7 c}, \Gamma_{8 c}$. In fact, in contrast to the band energies, the wave functions experience minor modifications under strain, and therefore the absolute values of the oscillator strenghts are nearly preserved. Following the trend with rising tensile uniaxial strain [21], an inversion of the two lowest conduction bands occurs, i.e., the $\Gamma_{7 c}$ conduction band appears below the $\Gamma_{8 c}$ band at $\Gamma$. This band inversion leads to huge oscillator strengths for optical transitions to or from the lowest conduction band, similarly to what is shown in Fig. 3 for $2 \mathrm{H}-\mathrm{Ge}$ and the second conduction band. A similar band inversion $\Gamma_{7 c} \Leftrightarrow \Gamma_{8 c}$ is suggested to happen in the band structure of the $4 \mathrm{H}$ polytype [3] of Ge [31]. Our own test calculations for $4 \mathrm{H}-\mathrm{Ge}$ do not give rise to such a band inversion nor, consequently, strong optical transitions to or from the lowest conduction band. This finding is in agreement with the fact that the pure hexagonal stacking in $2 \mathrm{H}$ is mixed with a cubic stacking in the $4 \mathrm{H}$ polytype [3].

\section{CONCLUSIONS}

In summary, we have clearly demonstrated by means of first-principles calculations that lonsdaleite germanium underlies a transition from a pseudo-direct semiconductor with a dipole-allowed but very weak optical oscillator strength into a direct semiconductor with typical strength of a $p \rightarrow s$ transition, upon perturbation of the ideal hexagonal lattice. Here we consider the substitution of one $\mathrm{Ge}$ atom with a Si atom in the four atom unit cell. The alloying of $2 \mathrm{H}-\mathrm{Ge}$ with $\mathrm{Si}$ leads not only to an increase of the interband transition energies but also to the enhancement of the dipole-matrix elements of all opti- cal transitions from the three highest valence bands into the lowest conduction band, independently of light polarization. The increase of the oscillator strengths at $\Gamma$ leads to matrix elements of the order of those for transitions involving the second conduction band. Strong oscillator strengths of the lowest interband transitions also occur in uniaxially strained $2 \mathrm{H}-\mathrm{Ge}$ because of the order inversion of the two lowest conduction bands. All in all, we conclude that perturbed lonsdaleite Ge is an excellent material for active optoelectronic applications in light-emitting diodes and lasers.

\section{COMPUTATIONAL METHODS}

The atomic configurations and lattice constants of the hexagonal crystals are optimized applying density functional theory (DFT) as implemented in the Vienna Ab-initio Simulation Package (VASP) [32] using the projector-augmented wave method [33] and a plane-wave cutoff of $500 \mathrm{eV}$. The shallow Ge3d electrons are treated as valence electrons. The modified Perdew-Becke-Ernzerhof (PBEsol) XC functional [34] is applied for structural relaxations. The BZ integration is performed using a $\Gamma$-centered $12 \times 12 \times 6 \mathbf{k}$-point grid. Atomic geometries are relaxed until the Hellmann-Feynman forces are below $1 \mathrm{meV} / \mathrm{A}$. The spin-orbit interaction is included for all electronic structure calculations.

\section{ACKNOWLEDGMENTS}

We acknowledge financial support from the H2020FETOpen projects SiLAS (grant agreement No. 735008) and OptoSilicon (grant agreement No. 964191).

\section{CONFLICT OF INTEREST}

The authors declare no conflict of interest.

\section{DATA AVAILABILITY STATEMENT}

The data that support the findings of this study are available on reasonable request from the corresponding author.

\section{KEYWORDS}

germanium, silicon-germanium alloys, lonsdaleite, electronic structure, optical matrix elements, light emission
[1] P. Ball, Nature 409, 1476 (2001), ISSN 1476-4687, URL https://doi.org/10.1038/35059301

[2] A. H. Atabaki, S. Moazeni, F. Pavanello, H. Gevorgyan, J. Notaros, L. Alloatti, M. T. Wade, C. Sun, S. A. Kruger, H. Meng, et al., Nature 556, 349 (2018), URL https://doi.org/10. 1038/s41586-018-0028-z.

[3] C. Raffy, J. Furthmüller, and F. Bechstedt, Phys. Rev. B 66, 075201 (2002), URL http://link.aps.org/doi/10. 
1103/PhysRevB.66.075201

[4] E. M. T. Fadaly, A. Dijkstra, J. R. Suckert, D. Ziss, M. A. J. van Tilburg, C. Mao, Y. Ren, V. T. van Lange, K. Korzun, S. Kölling, et al., Nature 580, 205 (2020), URL https ://doi . org/10.1038/s41586-020-2150-y

[5] C. Rödl, J. Furthmüller, J. R. Suckert, V. Armuzza, F. Bechstedt, and S. Botti, Phys. Rev. Materials 3, 034602 (2019), URL https://link.aps.org/doi/10. 1103/PhysRevMaterials.3.034602

[6] P. Borlido, J. R. Suckert, J. Furthmüller, F. Bechstedt, S. Botti, and C. Rödl, Phys. Rev. Materials 5, 114604 (2021), URL https://link.aps.org/doi/10. 1103/PhysRevMaterials.5.114604

[7] X. Cartoixà, M. Palummo, H. I. T. Hauge, E. P. A. M. Bakkers, and R. Rurali, Nano Letters 17, 4753 (2017), pMID: 28654293, https://doi.org/10.1021/acs.nanolett.7b01441, URL https:// doi.org/10.1021/acs.nanolett.7b01441

[8] N. Bao, F. Guo, D. Kang, Y. Feng, H. Wang, and J. Dai, Journal of Applied Physics 129, 145701 (2021), https://doi.org/10.1063/5.0043773, URL https://doi.org/ 10.1063/5.0043773.

[9] Z. Wang, Z. Zhang, S. Liu, J. Robertson, and Y. Guo, Applied Physics Letters 118, 172101 (2021), https://doi.org/10.1063/5.0044901, URL https://doi.org/ 10.1063/5.0044901.

[10] F. Bechstedt, Many-Body Approach to Electronic Excitations. Concepts and Applications (Springer-Verlag, Berlin, 2015).

[11] F. Tran, P. Blaha, and K. Schwarz, Journal of Physics: Condensed Matter 19, 196208 (2007), URL https://doi.org/ 10.1088/0953-8984/19/19/196208.

[12] F. Tran and P. Blaha, Phys. Rev. Lett. 102, 226401 (2009), URL https://link.aps.org/doi/10.1103/PhysRevLett. 102.226401

[13] A. D. Becke and E. R. Johnson, The Journal of Chemical Physics 124, 221101 (2006), https://doi.org/10.1063/1.2213970, URL https://doi. org/10.1063/1.2213970

[14] M. Laubscher, S. Küfner, P. Kroll, and F. Bechstedt, Journal of Physics: Condensed Matter 27, 405302 (2015), URL https: //doi.org/10.1088/0953-8984/27/40/405302

[15] P. Borlido, T. Aull, A. W. Huran, F. Tran, M. A. L. Marques, and S. Botti, Journal of Chemical Theory and Computation 15, 5069 (2019), URL https ://doi .org/10.1021/acs . jctc. 9b00322

[16] P. Borlido, J. Schmidt, A. W. Huran, F. Tran, M. M. A. L. Marques, and S. Botti, npj Computational Materials 6, 96 (2020), URL https://doi.org/10.1038/s41524-020-00360-0

[17] J. Heyd, G. E. Scuseria, and M. Ernzerhof, J. Chem. Phys. 118, 8207 (2003), URL http://link . aip.org/link/?JCP/ $118 / 8207 / 1$
[18] J. Heyd, G. E. Scuseria, and M. Ernzerhof, J. Chem. Phys. 124, 219906 (pages 1) (2006), URL http://link . aip.org/ link/?JCP/124/219906/1

[19] M. Gajdoš, K. Hummer, G. Kresse, J. Furthmüller, and F. Bechstedt, Phys. Rev. B 73, 045112 (2006).

[20] B. D. Malone, S. G. Louie, and M. L. Cohen, Phys. Rev. B 81, 115201 (2010), URL https://link.aps.org/doi/10. 1103/PhysRevB.81.115201.

[21] J. R. Suckert, C. Rödl, J. Furthmüller, F. Bechstedt, and S. Botti, Phys. Rev. Materials 5, 024602 (2021), URL https://link. aps.org/doi/10.1103/PhysRevMaterials.5.024602

[22] T. Inaoka, T. Furukawa, R. Toma, and S. Yanagisawa, Journal of Applied Physics 118, 105704 (2015), https://doi.org/10.1063/1.4930225, URL https://doi.org/ $10.1063 / 1.4930225$

[23] S. Mellaerts, V. Afanas'ev, J. W. Seo, M. Houssa, and J.-P. Locquet, ACS Applied Materials \& Interfaces 13, 30941 (2021), URL https://doi.org/10.1021/acsami.1c03700

[24] Sargent-Welch, Table of periodic properties of the elements (Skokie (Ill.), 1980).

[25] P. Tronc, Y. Kitaev, G. Wang, M. Limonov, A. Panfilov, and G. Neu, physica status solidi (b) 216, 599 (1999).

[26] F. Bechstedt and A. Belabbes, Journal of Physics: Condensed Matter 25, 273201 (2013), URL https://doi.org/ 10.1088/0953-8984/25/27/273201

[27] L. Gagliano, M. Kruijsse, J. D. D. Schefold, A. Belabbes, M. A. Verheijen, S. Meuret, S. Koelling, A. Polman, F. Bechstedt, J. E. M. Haverkort, et al., Nano Letters 18, 3543 (2018), URL https://doi.org/10.1021/acs.nanolett.8b00621

[28] A. Belabbes and F. Bechstedt, phys-

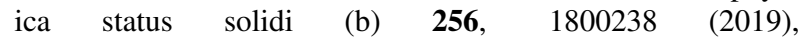
https://onlinelibrary.wiley.com/doi/pdf/10.1002/pssb.201800238, URL https://onlinelibrary.wiley.com/doi/abs/10. 1002/pssb. 201800238

[29] J. Greil, S. Assali, Y. Isono, A. Belabbes, F. Bechstedt, F. O. Valega Mackenzie, A. Y. Silov, E. P. A. M. Bakkers, and J. E. M. Haverkort, Nano Letters 16, 3703 (2016), URL https : //doi . org/10.1021/acs.nanolett.6b01038

[30] L. Gagliano, A. Belabbes, M. Albani, S. Assali, M. A. Verheijen, L. Miglio, F. Bechstedt, J. E. M. Haverkort, and E. P. A. M. Bakkers, Nano Letters 16, 7930 (2016), URL https : //doi.org/10.1021/acs.nanolett.6b04242

[31] F. Kiefer, V. Hlukhyy, A. J. Karttunen, T. F. Fässler, C. Gold, E.-W. Scheidt, W. Scherer, J. Nylén, and U. Häussermann, J. Mater. Chem. 20, 1780 (2010).

[32] G. Kresse and J. Furthmüller, Phys. Rev. B 54, 11169 (1996).

[33] G. Kresse and D. Joubert, Phys. Rev. B 59, 1758 (1999).

[34] J. P. Perdew, A. Ruzsinszky, G. I. Csonka, O. A. Vydrov, G. E. Scuseria, L. A. Constantin, X. Zhou, and K. Burke, Phys. Rev. Lett. 100, 136406 (2008), URL https://link.aps.org/ doi/10.1103/PhysRevLett.100.136406. 